$\begin{array}{cl}\begin{array}{cl}\text { Revue } \\ \text { de ihistoire } \\ \text { des religions }\end{array} & \text { Revue de l'histoire des religions } \\ & \begin{array}{l}1 \mid 2015 \\ \text { Genève, refuge et migrations (XV|e-XVII e siècles) }\end{array}\end{array}$

\title{
Refuge et migrations à Genève au miroir de polémistes, missionnaires et voyageurs $\left(\mathrm{XVI}^{\mathrm{e}}-\mathrm{XVII}{ }^{\mathrm{e}}\right.$ siècles)
}

Refuge and Migrations in Geneva in the Mirror of Polemicists, Missionaries and Travellers $\left(16^{\text {th }}-17^{\text {th }}\right.$ Centuries)

Daniela Solfaroli Camillocci

\section{(2) OpenEdition}

Journals

Édition électronique

URL : http://journals.openedition.org/rhr/8346

DOI : $10.4000 /$ rhr.8346

ISSN : 2105-2573

Éditeur

Armand Colin

Édition imprimée

Date de publication : 1 mars 2015

Pagination : 53-81

ISBN : 9782200929657

ISSN : 0035-1423

Référence électronique

Daniela Solfaroli Camillocci, « Refuge et migrations à Genève au miroir de polémistes, missionnaires et voyageurs (XV|e-XVII siècles) », Revue de l'histoire des religions [En ligne], 1 | 2015, mis en ligne le, consulté le 30 avril 2019. URL : http://journals.openedition.org/rhr/8346 ; DOI : 10.4000/rhr.8346 


\section{Refuge et migrations à Genève au miroir de polémistes, missionnaires et voyageurs $\left(\mathrm{XVI}^{\mathrm{e}}-\mathrm{XVII}^{\mathrm{e}}\right.$ siècles$)$}

Les témoignages de voyage présentent une lecture confessionnelle des usages de Genève et de son rayonnement international comme cité du refuge religieux. Les récits mettent cependant en lumière le lien complexe entre la ville et sa réalité territoriale, qui se modifie en fonction de la frontière politique, dépend de la dynamique socio-économique et des courants migratoires, et qui s'entretient par les relations familiales. Ils constatent finalement que ce lien trouble les barrières confessionnelles et entraîne des conséquences significatives sur le plan des croyances. Vers la fin de l'époque confessionnelle, les représentations polémiques de la migration religieuse et de la conversion servent ainsi un discours $d u$ retournement des apparences, qui met en question le lien établi entre lieux institutionnels et «bon choix» religieux.

\section{Refuge and Migrations in Geneva in the Mirror of Polemicists, Missionaries and Travellers $\left(16^{\text {th }}-17^{\text {th }}\right.$ Centuries $)$}

Travellers' narratives offer a confessional reading of Geneva's customs and of its international reputation as a city of religious refuge. However, these documents highlight the complex link between the city and its territorial reality, a relationship which changes depending on political boundaries, on socio-economic dynamics and on migration flows, and which is woven by family relationships. They eventually state that this link ends up blurring confessional boundaries and leads to significant consequences in terms of beliefs. At the end of the confessional age, polemical representations of religious migration and conversion also serve a discourse of the reversal of appearances that calls into question the link between institutional spaces and the "right" religious choice. 


\section{LA CITÉ DU REFUGE : LIEUX D'EXIL ET MYTHE HISTORIOGRAPHIQUE}

C'est parfois le hasard des routes qui amène à Genève le voyageur venant de France et se dirigeant vers l'Italie, l'Allemagne, les Pays-Bas, ou qui inversement se rend en France. Cependant, ce n'est jamais par hasard qu'un voyageur, arrivé à Genève, décide de s'arrêter et d'entrer dans la cité pour la visiter ou même d'y séjourner quelques jours. Les récits d'un tel détour ou séjour, qui témoignent d'un regard étranger posé sur la ville, sont, suivant le choix religieux du visiteur, loin d'être neutres ou anodins. Aux XVI ${ }^{\mathrm{e}}$ et $\mathrm{XVII}^{\mathrm{e}}$ siècles, les représentations de Genève sont en effet indissociables de son image confessionnelle, où l'organisation de l'Église réformée et la migration religieuse forment un tout. Les constructions discursives portant sur la ville comme le lieu par excellence du refuge religieux en Europe commencent peu après son passage à la Réforme, et introduisent une ambivalence agissant notamment sur le plan symbolique ${ }^{1}$. Genève est d'une part stigmatisée en tant que citadelle de bannis et d'apostats, qui apprêtent leurs armes pour détruire la chrétienté entière, comme le dénonce le prêtre parisien Artus Désiré dès la fin des années $1540^{2}$. La ville réformée est louée d'autre part, en raison de son ordre exemplaire et de son caractère de carrefour international. Comme l'écrit en 1558 un réfugié anglais renommé, John Bale, Genève est comparable aux grandes places commerciales, telles les villes de Londres, Paris et Francfort, mais elle est marquée par «une traffique nouvelle», celle des hommes et des femmes qui s'y rendent pour cause de religion ${ }^{3}$. Bref, dès la

1. Cf. Alain Dufour, «Le mythe de Genève au temps de Calvin», in Idem, Histoire politique et psychologie historique. Suivi de deux essais sur Humanisme et Réformation et le mythe de Genève au temps de Calvin, Genève, Droz, 1966, p. 63-95.

2. Artus Désiré, «Epistre au lecteur chrestien», Le Combat du fidelle papiste pelerin romain, contre l'apostat priapiste tirant à la synagogue de Genève maison babylonique des lutheriens, Rouen, Robert et Jean du Gort, 1550, cité par Francis Higman, «The origins of the image of Geneva», in J. B. Roney, M. I. Klauber (éd.), The Identity of Geneva. The Christian Commonwealth 1564-1864, Westport, 1998, p. 21-38.

3. John Bâle, Les vies des evesques et papes de Rome depuis la dispersion des Disciples de Jesus Christ jusques au temps de Paul quatrieme qui à present regne tyranniquement en l'Eglise, Genève, Conrad Badius, 1561, «Epistre» à Bullinger, 
moitié du siècle, la ville devient un «lieu exemplaire» de l'exil ${ }^{4}$, image à laquelle fait allusion une iconographie des Emblèmes de Théodore de Bèze illustrant la «vraie Église» par la silhouette d'une ville circulaire qui rassemble beaucoup à Genève $e^{5}$. La cité est représentée comme élevée dans les nuages et suspendue à un fil, donc isolée de son territoire - ce qui visualise à la fois sa condition réelle d'enclave et l'idée de l'incertitude vis-à-vis de son sort ultérieur. Le fil est cependant tenu par la main de Dieu - et l'idée de l'abandon confiant du fidèle à la volonté divine se superpose ici à celle d'une providence spécifique sur la ville (cf. fig. 1).

De ce «mythe de Genève» les chercheurs ont déjà tracé l'histoire, qui traverserait l'époque moderne, en transitant du religieux au politique et constituant le fondement d'un spécifique «esprit de Genève», pour aboutir finalement à la Genève des Nations ${ }^{6}$. Dans cette perspective historiographique, amplement redevable à l'histoire des idées, l'image positive ou négative de la ville serait le fruit d'une propagande religieuse et intellectuelle agissant notamment au niveau de l'idéologie et de l'imaginaire. La représentation de Genève, souvent stéréotypée, ne correspondrait que très partiellement à son évolution historique, à la réalité de la division politique de ses élites et des tensions sociales qui traversent les milieux artisanaux.

Rien de plus fréquent que d'opposer les «faits» découlant de la réalité sociale aux «constructions» de l'imaginaire. Cependant, les recherches sur l'histoire de la population à Genève à l'époque

Sulcer, Calvin, Melanchton, f. 14v ss., publiée partiellement par Théophile Dufour, «Bâle, Zurich et Genève en 1558. Fragment de lettre d'un Anglais», Mémoires et Documents publiés par la Société d'histoire et d'archéologie de Genève, t. 22, 1886, p. 10.

4. Sur la relation entre la construction théologique d'une vision «internationale» de la Réforme et les courants de la migration religieuse, cf. Heiko A. Oberman, «Europa afflicta: the Reformation of the Refugees» (1992), in Idem, John Calvin and The Reformation of the Refugees, Genève, Droz, 2009, p. 177-194.

5. Théodore de Bèze, Icones, id est verae imagines virorum doctrina simul et pietate illustrium [...] additis eorundem vitae et operae descriptionibus, quibus adjectae sunt nonnullae picturae quas emblemata vocant, Genève, Jean de Laon, 1580, f. [Ll.iiiv].

6. Cf. dernièrement Alfred Dufour, «Mythe de Genève et Esprit de Genève», Genève et la Suisse dans la pensée politiques: actes du colloque de Genève des historiens des idées politiques, éd. G. Busino, Aix-en Provence, Presse universitaires d'Aix-Marseille, 2007, p. 559-570, qui reconnaît sa dette à l'égard de l'article pionnier d'Alain Dufour, «Le mythe de Genève», op. cit. 
moderne et sur la relation entre refuge religieux et vie économique, montrent que les critères de réglementation du marché étaient aussi l'expression, au quotidien, d'un idéal religieux d'organisation sociale, et de la politique d'accueil des réfugiés pour cause de religion ${ }^{7}$. Par ailleurs, s'il est vrai que les courants des migrations dans la cité répondent à des besoins socio-économiques, comme pour toute ville de l'époque, il n'en reste pas moins que le développement spécifique de l'économie genevoise importée par les réfugiés français et italiens - la draperie et la soierie - a marqué durablement l'immigration régionale d'artisans et de salariés ${ }^{8}$. La politique d'accueil n'était toutefois pas sans susciter des réactions négatives, que ne manque d'exploiter la polémique religieuse. Dès la seconde moitié du XvI ${ }^{\mathrm{e}}$ siècle, les opposants de Calvin et de Bèze se chargent de dévoiler les arcanes d'un pouvoir qu'ils considèrent comme étant une véritable tyrannie théologique, dénonçant, comme le fait Jérôme Bolsec, la politique genevoise de l'accueil des réfugiés, liée selon lui moins à la cause de la religion qu'à la nécessité des réformateurs de soutenir leur réseau français. Leur stratégie épuiserait de cette manière les ressources économiques de Genève et appauvrirait ses habitants9 ${ }^{9}$ En fait, au cours des $\mathrm{XVI}^{\mathrm{e}}$ et $\mathrm{XVII}^{\mathrm{e}}$ siècles, crises alimentaires et épidémies de peste ont un caractère endémique dans la ville, suite aux guerres et à la fermeture des routes et des marchés vers la France. Il arrive qu'on attribue la responsabilité de ces difficiles conditions de vie aux orientations confessionnelles de la politique concernant les courants migratoires ${ }^{10}$.

7. Liliane Mottu-Weber, "Genève et ses minorités aux $\mathrm{XVI}^{\mathrm{e}}$ et $\mathrm{XVII}$ e siècles: l'apport des réfugiés réformés français et italiens», Il ruolo economico delle minoranze in Europa, secc. XIII-XVIII, Firenze, Le Monnier, 2000, p. 42-433.

8. Eadem, Economie et réfuge à Genève au siècle de la Réforme: la draperie et la soierie (1540-1630), Genève, Société d'histoire et d'archéologie de Genève, 1987.

9. Jérôme $\mathrm{H}$. Bolsec, Histoire de la vie, mœurs, actes, doctrine, constance et mort de Jean Calvin, jadis grand ministre de Genève, Paris, Gervais Mallot, 1577, f. 21r-22v; Idem, Histoire de la vie, mours, doctrine et déportements de Theodore de Bèze selon que l'on peut cognoistre jusqu'à maintenant en attendant que luy mesme si bon luy semble y adjouste le reste, Paris, G. Chaudière, 1582, f. $13 \mathrm{v}-14 \mathrm{r}$.

10. Liliane Mottu-Weber, «Le statut des étrangers et de leurs descendants à Genève ( $\mathrm{XVI}^{\mathrm{e}}-\mathrm{XVIII}^{\mathrm{e}}$ siècles)», Les immigrants et la ville. Insertion, intégraton, discrimination $\left(\mathrm{XII}^{e}-\mathrm{XX} \mathrm{X}^{e}\right.$ siècles), éd. Denis Menjot, Jean-Luc Pinol, Paris, L'Harmattan, 1996, p. 27-42; Eadem, «Genève et ses réfugiés. Politiques des 
Dès lors, le clivage entre la réalité et ses représentations est peut-être moins évident que ne le reconstruit rétrospectivement l'historien. Jusqu'à quel point l'image religieuse de la ville a-t-elle joué le rôle de filtre, en orientant à la fois le regard des témoins et l'attitude et les comportements des habitants et des magistrats ${ }^{11}$ ? Je souhaiterais aborder ici les représentations de Genève comme cité du refuge et ville de réfugiés, tout en modifiant l'angle d'observation privilégié par l'histoire des idées politiques. Les recherches sur la confrontation religieuse ont montré que le choix confessionnel s'affirme prioritairement par l'occupation symbolique des espaces urbains à travers les gestes publics et rituels, et qu'il est aussi rendu visible par une réorganisation des lieux susceptible de modifier le paysage ${ }^{12}$. Suivant ces perspectives d'étude, j'interrogerai pour ma part le processus de confrontation identitaire tel qu'il est représenté à travers des constructions discursives découlant d'expériences «de terrain », et qui proposent une lecture confessionnelle des usages de la ville, de sa dynamique socio-économique aussi bien que de son intégration dans la région ${ }^{13}$. Je commencerai par considérer l'image de Genève dans sa dimension locale pour revenir, à la fin de mon propos, à la représentation de son rayonnement international comme cité du refuge religieux.

autorités, réactions de la population $\left(\mathrm{XVI}^{\mathrm{e}}-\mathrm{XVIII}^{\mathrm{e}}\right.$ siècles $) »$, Migrations vers les villes: exclusion, assimilation, intégration, multiculturalité, éd. Hans-Jörg Gilomen, Anne-Lise Head-König, Anne Radeff, Zurich, Société suisse d'histoire économique et sociale, 16, 2000, p. 151-170.

11. Ma réflexion s'inspire ici de Roger Chartier, «Le monde comme représentation» (1989), in Idem, Au bord de la falaise. L'histoire entre certitudes et inquiétude, Paris, Albin Michel, 1998, p. 67-86.

12. Natalie Zemon Davis, «The Sacred and the Body Social in SixteenthCentury Lyon», Past and Present, 90, 1981, p. 40-70 et, plus récemment, Keith Luria, Sacred Boundaries. Religious coexistence and Conflict in Early-Modern France, Washington, The Catholic University of America Press, 2005; Christian Grosse, Les rituels de la cène: le culte eucharistique réformé à Genève (XVIXVII siècles), Genève, Droz, 2008; cf. aussi Alexandra Walsham, The Reformation of the Landscape: Religion, Identity and Memory in Early Modern Britain and Ireland, Oxford, Oxford University Press, 2011.

13. La référence complète des sources utilisées est proposée en annexe; elle est ici donnée en abrégé par le nom de l'auteur suivi de la date et de la page. 


\section{REGARDS SUR LA FRONTIÈRE CONFESSIONNELLE: LE TÉMOIGNAGE PERSONNEL}

Le dispositif de coexistence religieuse établi par les édits royaux qui mettent un terme aux guerres en France, change, à la fin du $\mathrm{XVI}^{\mathrm{e}}$ siècle, les modes de la confrontation entre catholiques et réformés. Le début des missions de l'intérieur qui s'attaquent aux frontières confessionnelles, en France comme dans le reste de l'Europe, paraît aussi modifier les visées et les stratégies du regard critique sur Genève, dont témoignent les livres de controverse tout comme les ouvrages apologétiques ou hagiographiques publiés dès 1598 . Parce qu'ils visent à une intervention des institutions politiques et/ ou religieuses pour affaiblir la position de l'Église adversaire, ces écrits déploient leurs arguments polémiques de manière de plus en plus ciblée; d'où la nécessité pour les auteurs d'intégrer des témoignages personnels, comme le fait par exemple Florimond de Raemond, et de les accréditer. C'est dans ce même but que Pierre Cayet reproduit des lettres et autres documents comme pièces à conviction ${ }^{14}$. À côté des informateurs politiques et des premiers missionnaires qui explorent la ville ${ }^{15}$, les convertis jouent évidemment dans la constitution du dossier sur Genève le rôle de témoins privilégiés d'un accueil et/ou d'une exclusion volontaire de la cité réformée ${ }^{16}$. Les documents analysés livrent des informations sur une période qui va des années 1580 aux années 1680, mais leur rédaction s'étale souvent sur plusieurs années, et les rédacteurs intègrent des sources différentes pour nourrir leur propos ${ }^{17}$. Dans ces récits, les représentations des courants migratoires intéressant

14. Raemond 1610, p. 932-937; Cayet 1599.

15. Le jésuite Luca Pinelli visite Genève en 1580 (Pinelli 1606); François de Sales visite Genève pour la première fois en 1595 , envoyé par l'évêque de Genève (résident à Annecy) Claude Grenier, dans le cadre des premières missions du Chablais (1594-1598); il a été suivi par des jésuites et des capucins, et notamment par Chérubin de Mauriane (qui se rend plusieurs fois dans la cité entre 1599 et 1602); les capucins Maurice de la Morrhe et Augustin d'Asti se rendent encore en mission à Genève en 1604 et 1605 (De Sales 1634, I, p. 129, p. 180-184; Charles de Genève 1653, II, p. 139-171). Giovan Francesco Biondi visite Genève lors d'un voyage en France en tant qu'informateur de la République de Venise (Biondi 1610). Je tiens ici à remercier beaucoup Chiara Petrolini, qui a attiré mon attention sur ce document inédit, dont elle m'a encore communiqué la transcription.

16. Cf. Cardoini 1621, Du Ranc 1609, Leti 1686.

17. Comme c'est le cas notamment de S. Locatelli (cf. infra l'analyse de son récit), qui visite Genève en mai 1665. La dernière version de ses mémoires de 
Genève correspondent en bonne partie aux visées spécifiques de leurs auteurs. Ces derniers entendent parfois produire une mémoire alternative des événements, parfois dénoncer des abus et solliciter une action répressive. Dans d'autres cas, ils cherchent à justifier et à défendre leur changement de confession ou racontent une expérience de voyage. Tous répondent néanmoins à la nécessité de légitimer leur processus d'écriture par leur expérience personnelle ou bien par l'emploi d'un témoignage d'autrui qu'on veut fiable ou « véridique» - fidèle à la réalité des choses - car découlant d'une expérience vécue et qu'on a pu vérifier. Il convient encore de souligner un élément, celui de l'intertextualité entre les imprimés polémiques ou de controverse portant sur Genève qui circulaient à l'époque et les récits personnels ${ }^{18}$.

\section{Arriver à Genève: la cité et Ses habitants}

Les récits montrent une certaine continuité en ce qui concerne l'analyse du dispositif urbain et l'observation des habitants. La première impression des visiteurs est celle du contraste entre l'aspect d'enclos de Genève - ville-citadelle élevée et protégée par des bastions et des murailles (cf. fig. 2) - et la facilité d'y accéder. Il y a en principe des gardes chargés d'enregistrer les entrées, cependant les voyageurs provenant des territoires catholiques peuvent entrer et traverser la cité sans problème; même les gens d'Église et les missionnaires n'ont pas besoin de rester incognito ou de s'habiller en séculier pour pouvoir y séjourner. Il s'agit là d'une donnée qu'on retrouve de la relation du jésuite Luca Pinelli, qui visite la cité en 1580, jusqu' au voyage de Sebastiano

voyage, qui ont circulé en plusieurs exemplaires manuscrits, date de 1693: Luigi Monga, «Introduzione», dans Locatelli 1691/93, p. 19-40.

18. Les témoignages des convertis et des témoins oculaires ainsi que les anecdotes des voyageurs sont employés dans les ouvrages polémiques (Cayet 1599; Raemond 1610), dans la biographie de François Sales écrite par son neveu (De Sales 1634), et dans l'historiographie missionnaire (Charles de Genève 1653); le publiciste G. Leti mentionne à plusieurs reprises la relation manuscrite du converti genevois A. Cardoini (Cardoini 1621; Leti 1686, III, pp. 145, 151-156). De leur côté, les mémoires personnels de Pinelli, de Cardoini et de Locatelli s'inspirent des images polémiques de Genève diffusées par les ouvrages de controverse. En général, il apparaît que tous les auteurs ont lu Bolsec et Raemond; Charles de Genève et Locatelli ont sûrement lu les hagiographies de F. de Sales. 
Locatelli, ecclésiastique italien en voyage de loisir, en $1665^{19}$. Il est donc relativement facile de visiter la ville. Les missionnaires capucins vont d'ailleurs exploiter cette facilité d'accès pour des actions de provocation: ils font leurs achats dans les boutiques, profitent des échanges pour nouer des contacts et pour diffuser des avis de prédication. Ils arrivent à obtenir le libre passage pour des pèlerins et des processions se rendant à Thonon, lieu à proximité de Genève, mais en territoire savoyard, lors des jubilés de 1602 et $1607^{20}$. Il est plus difficile, en revanche, de garder la liberté de mouvement. Les séjours de plus de trois jours sont interdits par les autorités; les visiteurs étrangers sont souvent pris en charge par leurs hôtes ou par des habitants, qui offrent leurs services comme guide, mais qui en fait suivent discrètement leurs mouvements et donnent l'impression de les contrôler ${ }^{21}$. Surtout, les portes de la ville peuvent se refermer rapidement et à l'improviste, en empêchant la sortie. C'est habituellement le cas afin de prévenir l'éventualité d'une agression armée lors des cultes qui rassemblent toute la population, mais les voyageurs qui se trouvent confrontés à ce dispositif de sécurité et qui sont obligés d'attendre pour sortir conservent quant à eux l'impression d'une ville-piège ${ }^{22}$.

Ce contraste entre l'ouverture et la fermeture de la ville se retrouve dans les remarques concernant le tempérament des habitants, dont on commence par observer invariablement la civilité et la courtoisie. La ville est tranquille et accueillante: il n'y a pas de bagarres dans les rues, les conversations sont détendues, les hôtes des logis, les commerçants se montrent bien disposés à l'égard des missionnaires ${ }^{23}$. Ce bon accueil fait aux gens d'Église en visite est d'ailleurs souligné dans un guide de Genève de Gregorio Leti,

19. Pinelli 1606, p. 134; Locatelli 1691/93, p. 290, 294.

20. Charles de Genève 1653, I p. 85-86; 107; 305-312; 390-391.

21. Pinelli 1606, p. 134-135; Du Ranc 1609, p. 32-34; 127-130; Locatelli 1691/93, p. 297; 299-301. Cardoini 1621, f. 100v note qu'un «Senatore» (il s'agit probablement d'un conseiller) aurait la charge de visiter une fois par jour les visiteurs provenant des pays catholiques (dans le cas notamment des «vassalli del papa, del re di Spagna, di Savoya, o d'altri Principi italiani»). De son côté, Leti témoigne avoir exercé ce rôle de guide pour les visiteurs étrangers et même avoir interrogé, à la demande de quelques ministres, des étrangers d'origine italienne ou espagnole qui avaient manifesté leur intention de se convertir, afin de vérifier leur bonne foi et conscience: Leti 1686, IV, p. 223-229.

22. De Sales 1634, II, p. 49; Locatelli 1691/93, p. 302.

23. Pinelli 1606, f. 94v. Charles de Genève 1653, II, p. 148-149. 
publié en traduction anglaise en 1681, peu après son départ de la ville ${ }^{24}$.

Mais les visiteurs catholiques découvrent vite que cette apparente disponibilité vise à discerner les intentions du visiteur, à examiner parfois sa foi ou qu'elle est le signe avant-coureur d'une discussion religieuse, voire d'une confrontation avec les autorités ${ }^{25}$. Les récits soulignent constamment le goût du peuple genevois pour la théologie. La controverse est ainsi présentée comme une pratique usuelle du débat dans les rues, comme en font l'expérience le jésuite Pinelli ou les missionnaires capucins. Ces derniers, entrés dans la ville vers 1604 sous prétexte d'acheter des livres, se retrouvent à débattre de religion dans les logis et les magasins, et suite à ces premiers échanges avec les habitants, sont suivis par des enfants et des femmes qui se rassemblent dans la rue pour surveiller leurs pas, en clamant qu'ils sont des «faux prophètes et des antichrists ${ }^{26}$. Une donnée qui revient dans ces témoignages, et qui est amplifiée par les pamphlets de controverse, est celle de l'aisance avec laquelle les femmes se rapportent aux livres sacrés ou discutent de religion ${ }^{27}$. Il y a des hôtesses qui demandent de vérifier dans la Bible les arguments du différend avec un visiteur ${ }^{28}$, des dames qui interrogent publiquement les missionnaires et d'autres qui n'hésitent pas à aborder des points doctrinaux ${ }^{29}$. Une autre donnée commune aux récits est celle de la représentation d'une ville largement redevable à la migration. Cité de réfugiés convertis, mais qui, en raison de sa condition d'enclave en territoire catholique, peut aussi abriter

24. Cf. Leti 1681 , p. 69 : «It is a common but false report in Italy that Monks, Friers (sic), Priests of the Church of Rome are badly used when they pass through Geneva, for they themselves find the contrary in their Travels, and if any man should offer insolence to a Priest, or Frier in that City, he would certainly be punished, nor do the people refuse Charity and lodging to the poor Religious when they demand it ». On retrouve ces affirmations aussi dans Leti 1686, IV, p. 630-635.

25. Cf. Pinelli 1606, f. 94r-v.

26. Charles de Genève 1653, II, p. 151-156.

27. Pinelli 1606, f. 89v.

28. Cayet 1599, f. Diiir.

29. Cf. Charles de Genève 1653, II, p. 153-154, où il rapporte que des dames genevoises interrogent les capucins tout en les défendant, «avec des coups de pieds et soufflets », des débordements des enfants rassemblés. Dans ce récit on peut lire de nombreux cas de femmes hérétiques interrogeant les missionnaires, et se montrant parfois émues par leur témoignage de vie exemplaire mais pas forcément convaincues par leur doctrine. Cf. encore ci-après la rencontre de Locatelli avec la genevoise Maria Pozzi. 
des saisonniers ou des commerçants provenant de la campagne et à l'identité confessionnelle moins nette, voire «papiste», Genève partage avec ses prosélytes, ses réfugiés ou migrants, l'image d'une ville à l'identité étrangère, celle-là même qui, suivant un informateur vénitien, rend difficiles, au début du XVII ${ }^{\mathrm{e}}$ siècle déjà, ses relations avec les Suisses $^{30}$. La dynamique sociale spécifique à Genève est bien visible dans son paysage: dans la deuxième partie du siècle, la cité semble «pleine comme un œuf», selon l'expression de Locatelli. Ce dernier, comme le jésuite Pinelli bien avant lui, se montre impressionné par la présence, massive à son dire, de défroqués, mais remarque que la sélection des réfugiés convertis et notamment des religieux apostats est particulièrement sévère. Il note ainsi que seuls les gens d'Église cultivés et les artisans expérimentés sont admis à y résider ${ }^{31}$.

Bref, l'expérience de Genève conforte les observateurs dans leur idée d'une société fondée sur la confession de foi, où le choix religieux investit chaque aspect de la vie quotidienne, forge la culture du peuple, marque l'économie de la ville et oriente la politique de ses dirigeants. L'image de la population de Genève ne correspond dès lors pas complètement à la représentation de l'hérétique qui abonde dans la littérature missionnaire de la frontière confessionnelle ${ }^{32}$. Dans son analyse polémique de cette «ethnologie protestante», elle oppose le peuple, potentiellement disposé à rentrer dans le giron de l'Église, à ses gouvernants et pasteurs, endurcis dans l'erreur. À Genève en revanche chaque habitant, y compris les femmes, parait se faire potentiellement missionnaire. Cette représentation d'une communauté solidaire dans sa croyance modifie partiellement l'image du libertinage des Genevois diffusée

30. Biondi 1610, f. 426r.

31. Locatelli 1691/93, p. 297-298; cf. aussi Pinelli 1606, p. 140 qui fait la connaissance de deux défroqués exerçant le métier de marchands de chapeaux. Leti 1686, III, p. 116-117 relève avec ironie la présence des défroqués comme un aspect caractéristique du refuge italien. Sur la question de l'accueil aux gens d'Église, cf. l'article de Nicolas Fornerod sur le dispositif de «discernement institutionnel» mis en place par la Compagnie des pasteurs de Genève pour les clercs catholiques réfugiés au début du XVII ${ }^{\mathrm{e}}$ siècle.

32. Cf. Bernard Dompnier, «Comprendre les stratégies catholiques de conversion dans la France du premier $\mathrm{XVII}^{\mathrm{e}}$ siècle», in Maria-Cristina Pitassi, Daniela Solfaroli Camillocci (éds.), Les modes de la conversion confessionnelle à l'époque moderne. Autobiographie, altérité et constructions des identités religieuses, Firenze, Leo Olschki, 2010, p. 191-213. 
par les premiers polémistes de l'époque de la Réforme, tels Désiré et Bolsec. Les récits soulignent plutôt la discipline des mœurs, l'intérêt excessif du peuple pour le fait religieux et son obstination: il s'agit d'une population de vrais faux-croyants. Par ailleurs, on peut bien se rendre à Genève depuis le territoire catholique pour y travailler ou pour échapper à des poursuites, mais il n'est possible d'y rester qu'à condition de se fondre dans l'organisation de la ville $^{33}$. Cependant, la sensibilité religieuse de la population agit à double tranchant. S'il est vrai qu'au premier regard des visiteurs, le choix confessionnel permet d'assurer un certain degré de conformité identitaire chez les habitants, et un encadrement social efficace, il n'en reste pas moins que les sources missionnaires et les récits de conversion sont en mesure de produire de nombreux cas de transfuges, destinés à rassurer le lecteur sur la fonction salutaire de l'Église catholique. Des adolescents, des femmes et de jeunes artisans avouent être impressionnés par les arguments soulevés par la prédication missionnaire, et se montrent insatisfaits de leur vie ou de leur organisation familiale, dont ils cherchent à s'échapper. Certains y arrivent parfois, d'autres renoncent; on souligne alors la difficulté à faire sortir les jeunes gens, la contrainte exercée par les familles, la cruelle autorité des pères ${ }^{34}$. Ces récits entendent finalement témoigner d'un malaise concernant la discipline sociale de la ville, dont la bonne disposition des jeunes et de certains ministres qui se convertissent fournirait la preuve ${ }^{35}$.

\section{VILLE, TERRITOIRE ET POPULATION}

La représentation des convertis qui s'évadent de la cité et se réfugient auprès des missionnaires installés dans les terres catholiques

33. Cardoini 1621, f. 114v-116r; Charles de Genève 1653, II, p. 190; Locatelli 1692/93, p. 298, 300.

34. De Sales 1634, I, qui rassemble de nombreux épisodes de conversion d'habitants de Genève de toute condition sociale; Charles de Genève 1653, II, p. 136, 148-151; 164-166; Charles de Genève 1657/80, p. 182-183.

35. Sur la question de la relation entre migration et conversion: Monica Martinat, «Conversions religieuses et mobilité sociale. Quelques cas entre Genève et Lyon au XVII ${ }^{\mathrm{e}}$ siècle», Mobilité et transmission dans les sociétés de l'Europe moderne, Rennes, Presses universitaires de Rennes, 2010, p. 139-157, ainsi que la contribution présentée ici. 
du duc de Savoie entourant Genève amène à la question du rapport entre la ville et son territoire. Cet aspect me semble en revanche se modifier dans les sources. Les premiers témoignages missionnaires sur la région livrent l'image d'une ville isolée, repliée sur elle-même et entourée d'un paysage désolé, comme le montre aussi un traité de controverse - déguisé en récit de conversion - publié par un capucin $^{36}$. Le frontispice reproduit d'un côté «L'Église de Dieu » - une ville s'élevant haut sur la montagne et aux toits bien garnis de croix - faisant face, de l'autre côté, au «déser [sic] d'invisibilité», la ville de Genève, encaissée dans une gorge surmontée par la silhouette menaçante de Calvin, et dont les toits sont totalement dépourvus de croix (cf. fig. 3).

Si le territoire autour de Genève est bien évidemment marqué par la guerre et constamment traversé par des armées, ce paysage paraît surtout, aux yeux des catholiques, dépouillé de tout signe évident de la foi, soit de croix, d'images sacrées, de lieux de culte appropriés $^{37}$. C'est donc une action à la fois de bonification visuelle de la région et de maintien de l'état d'isolation de la cité hérétique qu'entreprennent les missionnaires en employant la croix comme un ustensile-symbole. Les croix sont dès lors utilisées comme une manière de provocation, pour marquer les routes vers Genève et les chemins dans les forêts; elles sont amenées en procession autour de la ville, introduites dans les bastions lors du passage des pèlerins des paroisses catholiques se rendant à Thonon pour célébrer les jubilés, quoique couvertes afin de respecter les consignes des autorités ${ }^{38}$. Dans le même temps, les habitants sont attirés hors de la ville par les stratégies sonores et gestuelles des liturgies et des prédications improvisées lors de l'installation des croix, et les ministres sont défiés à des disputes théologiques, en territoire catholique, autour des bienfaits de la Sainte Croix. Les vertus salutaires du signe de la croix, seul véritable moyen de se protéger des diables qui infestent les campagnes et hantent les maisons, en effrayant les habitants, sont aussi exaltées par le discours missionnaire s'appuyant sur

36. Marcellin de Pont-de-Beauvoisin, La piperie des ministres et fausseté de la religion prétendue, ensemble la vérité catholique recogneües par le Sr de Pasthée gentilhomme dauphinois, advocat au parlement de Grenoble, Lyon, Loys Muguet, 1618.

37. De Sales 1634, I, p. 96-97.

38. Charles de Genève 1653, I, p. 308-321, 321-333. 
des faits «véritables» et des anecdotes locales d'exorcismes et de conversions ${ }^{39}$.

Cependant, bien que les informateurs politiques témoignent du sentiment d'isolation des élites genevoises et des difficultés économiques de la ville suite à l'action politique de la Savoie, axée sur l'embargo, qui rend difficile l'accès aux marchés et la circulation des biens produits à Genève, et sur la censure ecclésiastique, qui touche l'industrie principale du livre ${ }^{40}$, les récits missionnaires constatent que le lien entre la cité et son territoire est difficile à dissoudre. Il s'agit moins de l'obstination des fidèles des communautés réformées rentrées sous la domination de la maison de Savoie - comme celle de Thonon, dont la persistance dans l'hérésie est mise en scène afin notamment de souligner les vertus héroïques des convertisseurs ${ }^{41}-$, que de la découverte du fait que la ville hérétique attire les habitants du territoire. En effet, les possibilités d'emploi et de formation scolaire amènent vers Genève des émigrants de tous niveaux sociaux. Il y a encore les contacts quotidiens et notamment ceux permis par les marchés. De plus, la frontière confessionnelle divise les familles mais ne coupe pas entièrement le réseau de solidarité sociale ni surtout les parentés ${ }^{42}$. Il est vrai que ces éléments agissent à double tranchant, et que pour les agents du duc de Savoie et les missionnaires il est relativement simple d'avoir des informateurs dans la ville. Reste que l'attrait exercé par la cité hérétique sur le territoire environnant est mis en lumière dans un mémoire, destiné à solliciter la fondation de la maison pour les convertis et celle de l'académie de Thonon en 1602, mémoire reproduit dans la biographie de François de Sales écrite par son neveu en $1634^{43}$. De par son contexte de publication, ce document transmet une représentation polémique de Genève assez paradoxale. Tout en étant

39. Charles de Genève 1653, II, p. 126, 260-61.

40. Biondi 1610, f. 426r-v.

41. De Sales, 1634, I, p. 150-232; Charles de Genève, 1653, I: la première partie de l'ouvrage est entièrement consacrée à la «conversion» du Chablais.

42. Comme l'explique un gentilhomme catholique du Chablais aux missionnaires capucins vers 1604-1605, «j'ay dans Genève des fort proches parentz et étroictement alliés et, nonobstant la diversité de religion, nous ne laissons pas de persévérer en bonne amytié avec eux et, dans les occasions de mariage et festins nuptiaux, nous nous invitons réciproquement et nous assamblons, sans toutefois nous démarcher de notre devoir en matière de religion »: Charles de Genève 1653, II, p. 146.

43. De Sales 1634, I, p. 143-147. 
négative, elle souligne néanmoins les aspects sociaux et matériels de la capacité d'attraction de la ville, mais également son prestige intellectuel.

Le danger mis en avant est que le peuple catholique du territoire s'inspire des usages des habitants hérétiques de la ville. Cet élément est relevé notamment par les missionnaires capucins, dont les écrits historiographiques réagissent implicitement à l'exaltation du rôle missionnaire de François de Sales mis en avant par ses hagiographes, au détriment de l'action des membres de leur ordre. L'historiographe de la mission permanente de Savoie retrace le dispositif des couvents capucins mis en place entre 1608 et 1623 autour de la ville en le représentant sous la forme d'une croix, qui dès lors tiendrait Genève «comme assiégée et, s'il est permis de le dire, crucifiée » (cf. fig. 4). Mais ce dispositif symbolique de sécurité n'arrive pas vraiment à empêcher le danger d'une «zone grise» de la croyance. Si l'historiographe capucin se déclare heureux des succès de l'action missionnaire aux alentours de Genève, il note néanmoins, encore pour les années 1640, que «ceux même qui tâchent de conserver les sentiments de la foi s'y corrompent si étrangement qu'on a peine de les discerner d'avec les hérétiques ». Les catholiques possèdent des livres interdits et ne vont pas à la messe, les paysans ne peuvent pas être détournés «d'aller servir et trafiquer sur les terres des hérétiques $»^{44}$. D'où l'attention portée par les missionnaires à la diffusion, parmi la population, des possessions diaboliques, et donc à la présence de frénétiques et de démoniaques dans les campagnes. Ce sont des phénomènes qui obligent les gens d'Église catholiques actifs sur la frontière à un travail inlassable d'exorcismes, les possédés leur étant aussi envoyés du lointain et, comme l'écrit Charles de Genève, à la suggestion même des ministres bernois. En constatant l'inefficacité de leurs prières de délivrance, ces derniers auraient conseillé aux familles des possédés de les présenter «aux capucins, car il se fait par-delà (scil.: à Thonon) un certain exercice des papistes quy conjurent les démons par l'entremise de leurs ecclésiastiques et en délivrent quelques-uns $»^{45}$.

44. Charles de Genève 1657/80, p. 182,186, 194, 201.

45. Charles de Genève 1653, II, p. 325; cf. encore De Sales 1634, I, p. 164, 414, qui souligne l'exorcisme comme tâche pastorale spécifique pour laquelle François de Sales a été sollicité tout au long de son épiscopat. 
Bref, ces récits mettent en lumière le lien complexe - sur le plan religieux -, entre la ville et sa réalité territoriale, lien qui non seulement se modifie en fonction des guerres et de la frontière politique, mais qui dépend encore en bonne mesure du tissu économique et des usages sociaux, flotte avec les courants migratoires des saisonniers, et s'entretient par les relations de parenté. Derrière ces représentations du quotidien religieux autour de Genève affleure le constat que ce lien trouble les barrières confessionnelles et entraîne des conséquences significatives sur le plan des croyances. Dès les années 1640 , les sources missionnaires, tout en visant à légitimer la nécessité d'une présence accrue des missions dans le territoire, semblent témoigner d'un travail de deuil de cette existence inévitable de la ville, en assurant qu'elle est due moins à son Église qu'aux raisons contraignantes de la politique et aux intérêts matériels d'une population rurale, qui paraît parfois insouciante du salut de son âme.

\section{DisSONANCES: LES LIMITES CONFESSIONNELLES À L'ÉPREUVE}

Le discours polémique, compris en tant que construction réaménageant l'expérience du terrain, reflète un mode d'appropriation d'un espace confessionnel et de sa dynamique religieuse spécifique par le biais de son interprétation à l'intérieur de catégories «collectives », établies et communes tant à l'écrivain qu'à son lecteur, y compris un lecteur critique ${ }^{46}$. Cependant, ce processus comporte aussi des dissonances. Tout comme chaque récit de conversion appelle potentiellement des narrations différentes qui s'y opposent - il y a autant de réfugiés que de transfuges -, l'expérience de Genève en tant que ville marquée par la migration religieuse interpelle les témoins. C'est pourquoi ce mouvement d'individus doit être expliqué et justifié sur le plan confessionnel par les acteurs institutionnels ${ }^{47}$ - et cela indépendamment des raisons individuelles

46. J'emploie ici la notion d' «appropriation» suivant la (re)formulation de Roger Chartier, op. cit., p. 73-74.

47. Pour cette question, cf. Thierry Wanegffelen, «Se convertir ou abjurer? Indices de la construction confessionnelle dans les cérémonies et adhésion aux Églises réformées et catholiques en France au $\mathrm{XvI}^{\mathrm{e}}$ siècle», Catéchismes et confessions de foi, Actes du VIII ${ }^{e}$ colloque Jean Boisset, éd. Marie-Madeleine Fragonard et Michel Peronnet, Montpellier, Université Paul Valéry, 1995, p. 65-93. 
des conversions. La multiplicité des cas peut finalement engendrer des représentations contradictoires du choix confessionnel. $\mathrm{Au}$ cours du XVII ${ }^{\mathrm{e}}$ siècle, le courant alternatif d'émigration/immigration, qui marque les dimensions régionale et internationale de Genève, questionne de plus en plus ses observateurs.

Le récit du voyageur italien Sebastiano Locatelli dévoile que l'adaptation du regard personnel au témoignage confessionnel peut s'avérer difficile, ou bien ambivalente. Ce jeune prêtre bolonais emprunte la route de Genève en 1665, au retour d'un séjour en France où il a accompagné des aristocrates en voyage de loisir. La halte des voyageurs est courte, trois jours à peine, suivant le dispositif de l'accueil des étrangers à Genève, mais cela suffit à Locatelli pour tracer un portrait détaillé de la ville dans son journal de voyage. Il s'intéresse à tous les aspects de la vie quotidienne dans «ce paradis de pestiférés de l'hérésie de Calvin et de religieux apostats, paradis qui cependant prend fin le premier jour de la maladie et le dernier de la vie $»^{48}$. Il décrit les fortifications, l'hôtellerie, les marchés et les boutiques, les lieux de culte, l'hôtel de ville, demande à ses guides de le renseigner sur l'organisation politique, pose des questions sur le dispositif institutionnel de l'accueil aux réfugiés. Deux aspects de la vie locale polarisent toutefois son attention, la population féminine et les usages religieux - et l'une ramène sans cesse aux autres.

Chez Locatelli, la rhétorique de l'érotomanie, avec la mise en scène de l'échec sexuel - traits caractéristiques de son journal de voyage -, peuvent être lues comme une manière de la médiation culturelle. Défiant les limites de la communication lors des échanges préliminaires du jeu érotique, le désir contourne les difficultés linguistiques, et transforme la confrontation en rencontre, les usages différents en pluralitét ${ }^{49}$. Dans le souvenir de sa visite de Genève,

48. Locatelli $1691 / 1693$, p. 289. Ici et après, les traductions du texte sont miennes. Je tiens à remercier Maria-Cristina Pitassi de l'aide qu'elle m'a apportée pour résoudre les problèmes de traduction de certains passages. - Cf. aussi la traduction en français, établie sur un seul manuscrit, mais bien documentée: Voyage de France. Moeurs et coutumes françaises (1664-1665). Relation de Sébastien Locatelli prêtre bolonais, traduite sur les manuscrits autographes et publiée avec une introduction et des notes par Adolphe Vautier, Paris, Picard et fils, 1905.

49. Sur cet aspect de l'écrit de Locatelli je renvoie à l'article très stimulant de Paul Cohen, «The women in Father Locatelli's linguistic life: an italian priest's travels and the erotics of linguistic plurality in Early Modern Europe», dans Worth 
Locatelli joue souvent de l'effet de surprise, par l'alternance dans son discours des registres comique et moral. Le lien qu'il noue entre désir sexuel, frustration et religion, lui permet finalement de produire un témoignage qui met en œuvre une construction littéraire de la conversion religieuse en tant que 'retournement' imprévu suite au dévoilement des apparences trompeuses se cachant derrière la logique rassurante de la confrontation confessionnelle.

Le soir de son arrivée à Genève, Locatelli et ses compagnons font connaissance, dans une épicerie, de sa très charmante patronne, Maria Pozzi, qui leur raconte être la fille d'une nonne et d'un religieux apostats d'origine milanais, et la femme d'un épicier juif converti, Benjamin Rubatti ${ }^{50}$. Au cours de leur conversation, Maria se montre désireuse d'aborder le sujet de la religion. Ainsi le jeune prêtre, «se rendant à son désir», tout en en craignant les conséquences, lui pose des questions sur la doctrine de Genève, que Maria expose avec aisance à son auditoire sidéré ${ }^{51}$. De cet échange Locatelli sort, comme il l'avait pressenti, perdant. Tout d'abord, il déclare avec malaise de ne pas pouvoir noter dans son journal les arguments théologiques mis en avant par Maria, qu'il considère très efficaces pour le commun peuple et donc dangereux pour un lecteur potentiel non averti. Il est encore obligé de reconnaître que la préparation théologique de la jeune hérétique lui paraît supérieure à celle d'un curé catholique ordinaire - affirmation qui n'est pas sans malice, à la fois anticléricale et auto-ironique. Surtout, il doit avouer que cette première rencontre nocturne éveille chez lui le désir de mieux connaître les usages religieux de Genève.

Les jours suivants, Locatelli visite la cité, assiste à la prédication d'un prêtre apostat de renom - Jean de Labadie -, dont Maria lui a parlé, explore les lieux de culte, interroge des défroqués. Les informations qu'il reçoit sur la sévérité de la réglementation présidant à leur sélection ne sauraient encourager l'apostasie. Locatelli note plusieurs anecdotes sur les peines imposées pour toutes sortes d'infractions à la discipline de la part de certains moines réfugiés

and Repute: Valuing Gender in Late Medieval and Early Modern Europe. Essays in Honour of Barbara Todd, Kim Kippen and Lori Woods éd., Toronto, CRRS Publications, Victoria University, 2011, p. 391-429.

50. La boutique du «spetial Rubbati » est mentionnée aussi par Leti comme un lieu de Genève propice à l'échange de nouvelles: Leti 1686, IV, p. 254.

51. Locatelli 1691/93, p. 290-293. 
mal avisés, mais souligne également, pour le blâmer aussitôt après, le bon témoignage de vie de ceux qui restent et, plus généralement, de cette population de convertis «scélérats». Certains convertis expriment avec liberté leurs opinions. Un médecin expérimenté de Modène, un juif devenu chrétien, que Locatelli a connu catholique à Lyon, et qu'il retrouve à Genève en fidèle réformé, affirme que «celui qui n'est pas bon juif, n'est non plus bon chrétien (scil.: catholique), dès lors il est mieux qu'il devienne hérétique, car cela revient au même ${ }^{52}$. De telles sortes de propos mécréants, Locatelli assure en avoir entendus encore par son hôte, un autre juif converti, qui de son côté a choisi de passer directement à la Réforme et déclare, en plaisantant, aux visiteurs: «qui n'est pas bon juif, ne sera jamais bon chrétien. Ne serait-ce, Messieurs, que, n'ayant pas su vivre en bon juif, cette fois-ci je finisse par devenir bon calviniste !» L'hôte se plaît encore à argumenter que «si vos théologiens disent que si peu de chrétiens seront sauvés, du fait qu'on sera obligé d'aller à la maison du Diable autant y aller pour quelque chose qui en vaille la peine plutôt que pour des bagatelles, tel - j'en suis convaincu - le fait de ne pas avoir respecté le précepte du jeune avant la fête $»^{53}$.

Si l'impiété de ces juifs convertis n'est pas en soi une surprise pour Locatelli, celui-ci se montre en revanche impressionné par le style de vie de quelques religieux apostats, selon lui destinés sans doute à la damnation, mais qui justifient le bien-fondé de leur choix par leur vie conjugale épanouie, dont témoigne leur nombreuse progéniture. Avec ces convertis qui exhibent avec fierté leur famille et leur bonne intégration sociale, l'autre découverte surprenante de Genève - le charme de sa population féminine - inspire de nombreux commentaires. Le regard de Locatelli est tout d'abord envoûté par les belles paysannes du marché, qu'il découvre toutefois catholiques, car provenant des territoires ruraux environnant la ville. Quant aux magasins de la cité, l'usage qu'il observe de se servir de jeunes femmes pour attirer les clients, ne diffère guère de la pratique répandue parmi les Français ${ }^{54}$. De leur côté, les femmes de Genève sont belles et fort aimables, apprécient le luxe, sont parées de bijoux et exhibent des bagues à tous les doigts. Elles suscitent

52. Ibid., p. 297.

53. Ibid., p. 301.

54. Ibid., p. 296. 
l'admiration ironique des voyageurs en tant qu'elles sont «très modestes» se rendant au temple avec leurs riches toilettes, mais Locatelli découvre vite que si elles le regardent et le saluent, avec la plus grande déférence, c'est uniquement parce que sa robe courte rassemble à celle portée par les ministres. Cette bonne disposition des Genevoises lui est confirmée par une deuxième rencontre avec Maria Pozzi, le jour même de son départ. Locatelli la croise alors qu'elle se rend au temple - joliment parée de boucles, bijoux et rubans - pour participer au culte de la Pentecôte. En apprenant de la belle épicière qu'elle prend part à la Cène, Locatelli est saisi par le désir irrésistible de lui lancer un défi érotico-confessionnel. Se jetant à son cou et lui donnant un baiser sur la bouche, il déclare à haute voix, d'un ton de provocation, qu'elle peut aller sans autre communier, maintenant qu'un prêtre papiste l'a embrassée... Mais Maria promptement lui répond, en souriant, qu'elle pourra communier sans scrupule, tandis que les prêtres papistes, tel que lui, vont à l'autel pour manger le corps et boire le sang de Jésus après avoir couché la nuit avec leur concubine. La riposte de la jeune femme tient lieu ici du coup de théâtre. Bouleversé par le souvenir de cet échange, Locatelli coupe dramatiquement son récit pour faire appel à son lecteur, dont il devine l'horreur morale, en lui demandant de prières impétrant pour lui le pardon de ce qu'il reconnaît être le pire des péchés dont il s'est rendu coupable dans son voyage. Ce baiser, qu'il a essayé de justifier dans son âme comme un geste de mépris à l'égard du «feint sacrement» des hérétiques, lui a été en réalité inspiré par la beauté admirable du visage de la jeune hérétique. En reprenant le fil de son histoire, Locatelli conclut que Maria reprend sa route vers le temple, en laissant derrière soi les visiteurs intimidés, et l'auteur du récit plongé dans sa honte ${ }^{55}$.

Cet épisode, dont le caractère anticlérical est souligné par le changement subit de registre, du comique au moral, est rapporté dans un seul des manuscrits du Voyage de Locatelli qui nous sont parvenus, l'une des premières rédactions datant de 1675. Dans les versions successives, l'anecdote est censurée par l'auteur, qui lui substitue une ample digression où il décrit la liturgie de la Cène réformée de Genève à laquelle il a finalement assisté, description qu'il fait précéder d'une réfutation préalable de la doctrine 
calviniste de l'eucharistie sous la forme d'une controverse fictionnelle ${ }^{56}$. Dans cette défense de la transsubstantiation face à un public imaginaire de calvinistes, Locatelli joue le rôle principal de champion catholique - en prenant ainsi sa vengeance, a posteriori et inavouée, sur Maria Pozzi. Le baiser volé, troublant la frontière entre hérésie et péché, d'acte érotique, et donc de faute morale, devient ainsi l'instrument implicite de la mise en scène d'une conversion religieuse imprévue, celle de l'auteur du récit ${ }^{57}$. À la fin de son séjour genevois, Locatelli se montre convaincu de la nécessité salutaire du témoignage apologétique. Comme il le déclare au lecteur, sa digression théologique a le caractère d'un «débordement spirituel», d'une réaction à son expérience de la ville hérétique. La controverse fictionnelle qui conclut la visite de Genève correspond ainsi à un récit de conversion, celui du voyageur désengagé, voire sceptique, «converti» au débat théologique. En fait, cette controverse n'aurait jamais pu se produire à l'époque de son voyage, en raison notamment de son incompétence - manifeste et déclarée - en matière de doctrine. Mais avec un dernier clin d'œil, qui dévoile le caractère libertin de cet écrit, Locatelli rassure enfin son lecteur. Il n'a pas l'intention de faire de longs sermons, puisque de toute manière il ne s'agit pas d'arguments qu'il professe. Dans l'exposé, il s'est limité à reprendre les motifs des controverses entre catholiques et huguenots auxquelles il a souvent assisté à l'époque de son voyage à Paris, puisque «comme je l'ai écrit en plusieurs endroits, je n'ai jamais voulu éprouver mes forces contre les hérétiques, en raison de mon extrême ignorance $»^{58}$.

56. Ibid., p. 303-306.

57. Dans la dernière version de son Voyage, Locatelli ajouta néanmoins un passage renvoyant à l'anecdote censurée du baiser volé, qui est alors présenté comme une felix culpa. Il rapporte qu' après le départ de Genève, le récit burlesque des adieux à Maria Pozzi fait aux absents par les témoins qui se moquent de l'intempérance de leur compagnon, froisse Agnese, une jeune femme qui fait partie de la compagnie. L'inimitié qu'elle lui manifeste jusqu'à la fin du voyage, éloigne l'éventualité de quelques «tentations majeures » à l'égard d'une compagne de voyage pour laquelle Locatelli avoue son inclination, d'où le commentaire concernant le caractère providentiel de son geste inconsidéré vis-à-vis de la «belle hérétique» (ibid., p. 308).

58. Ibid., p. 306. Locatelli rapporte des «disputes de religion» qui avaient lieu «toujours après le déjeuner et le dîner» dans l'hôtel de Paris où séjournait le groupe (p. 195). Pour une lecture en partie différente du lien établi par Locatelli entre érotisme et religion, qui nuance la dimension libertine de son journal de voyage, cf. Paul Cohen, op. cit., p. 418-422. 


\section{DÉNONCIATIONS : LES ARCANES DE LA FRONTIÈRE RELIGIEUSE}

Parce qu'elles se prêtent au décentrement du regard, les représentations polémiques de la migration religieuse et du changement de confession peuvent servir le processus libertin de retournement. C'est ce que montre encore le témoignage sur Genève de Gregorio Leti, publié dans les années de crise confessionnelle qui suivent la Révocation ${ }^{59}$.

En 1679, après vingt ans de séjour à Genève, ce publiciste d'origine italienne, qui pendant sa jeunesse avait fait l'expérience du refuge et de la conversion au calvinisme, et qui avait désormais obtenu la bourgeoisie de la ville, est obligé d'abandonner la République en raison de son activité éditoriale, que la Compagnie des pasteurs juge indigne d'un réformé. Accusé de cryptocatholicisme et d'immoralité, Leti se réfugie en France, mais il refuse à Paris de rentrer dans le giron de Rome comme on le lui demande, et se rend en Angleterre. Obligé de partir, toujours en raison de ses publications jugées médisantes, il termine ses jours à Amsterdam, où il adhère à l'Église wallonne, donc à la communauté de l'orthodoxie réformée. Dans son Historia genevrina, un ouvrage en cinq volumes publié en 1686, Leti reconstruit l'histoire de Genève de l'époque ancienne à ses jours, en s'arrêtant notamment sur la politique locale. Il entend montrer que les raisons de la survie de cette petite enclave protestante en territoires catholiques, qui a l'apparence d'un miracle, sont moins d'ordre providentiel que géopolitique. En mettant en avant son expérience personnelle, Leti démystifie tout d'abord l'image de Genève découlant de la polémique catholique, dont il ridiculise les faussetés et les naïvetés ${ }^{60}$. Il critique néanmoins les excès commis à l'époque de la Réforme par les réformateurs, dont il ne trace pas un portrait élogieux ${ }^{61}$. En arrivant au récit de son temps, Leti

59. Je me permets de reprendre ici, en les développant, les conclusions de mes articles sur le séjour genevois de Leti, auxquels je renvoie aussi pour la bibliographie sur cet auteur: "L'activité éditoriale de Gregorio Leti à Genève, entre libertinisme et tradition polémique (1661-1679) », Libertinage et Philosophie au XVII siècle, 8: Protestants, hérétiques, libertins, Saint-Étienne, Publications de l'Université de Saint-Etienne, 2004, p. 47-69; «Un regard libertin sur la Réforme et les réformateurs: la fin controversée du séjour de Gregorio Leti à Genève», in Léonard Burnand, Adrien Paschoud (éds.) Espaces de la controverse en France au seuil des Lumières (1680-1715), Paris, Champion, 2010, p. 97-122.

60. Leti 1686, I, p. 6-10.

61. Ibid., II, p. 489 ss.; III, p. 57 ss. 
veut témoigner d'un réel malaise social et intellectuel à l'intérieur de la ville, dû à l'endurcissement du contrôle sur les opinions religieuses, et notamment sur celles théologiques concernant la grâce, qu'il dénonce comme une conséquence de l'intolérance des théologiens de l'Académie et du zèle excessif de certains ministres. La censure dont il a lui-même été victime ainsi que le procès qui est à l'origine de son départ et dont il dévoile dramatiquement tous les arrière-plans, ne seraient dès lors qu'un exemple ultérieur de cette dégradation des coutumes ${ }^{62}$.

Cette méthode d'enquête, qui fait constamment appel au décentrement critique du regard de la culture politique libertine dont Leti s'inspire, est encore employée dans l'analyse de la position de Genève à l'intérieur du réseau international réformé, qui est à l'époque déchiré par les polémiques sur les conséquences des persécutions des huguenots en France. De son côté, Leti blâme la politique d'intolérance de Louis XIV, qu'il déclare admirer comme roi mais qu'il juge mal conseillé en cela par les gens d'Église qui lui sont proches; dans le même temps, il démasque dans ses conclusions la politique du gouvernement genevois, qu'il considère ambivalente vis-à-vis du Refuge. En effet, la nécessité idéologique de tenir son rôle de «ville-mère» du calvinisme par une politique ample d'accueil, pourrait étrangler Genève sur le plan social et économique, en raison de sa petite dimension territoriale. Dès lors, quoique les autorités se cachent derrière l'excuse de l'embargo catholique qui les empêcherait de faire face aux réfugiés, Leti admet l'éventualité qu'un accueil sans conditions des persécutés ne corresponde pas à leurs orientations politiques actuelles ${ }^{63}$. Ce qui dès lors est mis en avant - implicitement - est que les raisons historiques de l'accueil donné à Genève aux réfugiés pour cause de religion sont moins inhérentes au soutien de la Réforme qu'aux besoins sociologiques et économiques de la ville ${ }^{64}$.

C'est finalement par la mise en scène de son expérience du terrain, et de son statut de converti ou de transfuge, que Leti dénonce les

62. Ibid., V, p. 242 ss.

63. Ibid., p. 484-487.

64. Avis que Leti exprime déjà dans son guide de Genève, cf. Leti 1681, p. 68 : «They were accustomed for a long time to receive and protect all that came and embraced the Reformed Religion, but at present the City being well peopled, they are not so ready to admit of such». 
logiques confessionnelles, qu'il considère comme le produit d'un zèle religieux dangereux pour le bien-être social. Le souci de vérité de l'historien en tant qu'observateur critique d'une actualité en devenir - qu'il faut nécessairement décrypter de l'intérieur pour pouvoir la comprendre -, se pose dans cet ouvrage comme le critère légitimant un témoignage qui ne sert plus la controverse théologique. Se trouve ainsi réfutée toute vision apologétique - que Leti juge en soi «passionnée» - qui est sous-jacente à l'obligation du converti de produire un récit spirituellement engagé de son expérience.

Dans mon analyse, j'ai essayé de montrer comment, tout au long de l'époque confessionnelle, les récits de Genève amplifient l'idée d'une «vocation» urbaine spécifique. Celle-ci peut être décelée par la manière dont elle inspire les comportements des habitants, marque durablement leur tempérament, influence, voire modifie le rapport entre la cité et son territoire, et parvient parfois à s'imprimer dans le paysage lui-même. À l'époque de la Réforme, le processus polémique de renversement, qui définit des réalités communautaires en les représentant comme antithétiques, a contribué amplement à la définition des oppositions confessionnelles. Dans les constructions discursives découlant de l'expérience de la confrontation religieuse, ce même processus de renversement est employé afin de représenter la solidité des barrières confessionnelles, qui sont dès lors présentées comme des réalités effectives et visibles agissant au niveau des appartenances identitaires et territoriales. Dans ce contexte, la mise en question critique d'une notion de «frontière» religieuse obéissant à une stricte logique confessionnelle surgit moins de l'éloignement intellectuel de la culture de la controverse que de l'usage détourné des images dissonantes que la polémique religieuse a elle-même engendrées - parfois malgré elle, parfois à des fins spécifiques -, telle la représentation des bonnes mœurs des Genevois-e-s ou des mauvaises coutumes religieuses des habitants des territoires catholiques dans les écrits des missionnaires. Ainsi, dans le témoignage de Locatelli, c'est la réaction vertueuse de la femme hérétique qui, tout en rétablissant les limites de la bienséance confessionnelle, dévoile à la conscience du voyageur catholique la tentation cachée derrière le geste provocateur apparemment inspiré par une intentionnalité apologétique. De la même manière, par sa critique de l'image protestante de Genève comme ville du refuge, 
critique qui se veut impartiale, car découlant d'un parcours de vie «transconfessionnel» dans l'Europe des confessions, Gregorio Leti produit une écriture historique sur les migrations qui entend vider la frontière religieuse de ses raisons d'être. En exploitant la rhétorique du retournement des apparences, Locatelli et Leti invalident moins les raisons spirituelles de la conversion, que l'efficacité du lien établi entre lieux institutionnels et 'bon choix' religieux.

daniela.solfaroli@unige.ch 
ANNEXE I

\section{DOCUMENTS CITÉS, DANS L'ORDRE CHRONOLOGIQUE}

\section{MANUSCRITS}

Pinelli 1606: Luca Pinelli, «Alcune cose più notabile e pericoli accaduti a me Luca Pinelli della compagnia di Giesu»(1560-1606), Roma, Archivum Romanum Societatis Jesu, fondo gesuitico, ms. 2 to. 2 Miscellaneum 2-II, f. 85r-99r (publié partiellement in Mario Scaduto, «La Ginevra di Teodoro Beza nei ricordi di un gesuita lucano, Luca Pinelli (1542-1607)», Archivum Historicum Societatis Jesu, XX (1951), p. 117-142).

Biondi 1610: Giovan Francesco Biondi, «Discorso sopra le cose avvenute et considerate da me coll'occasione del mio viaggio in Delfinato nell'anno 1610», London, Public Record Office State Papers Foreign, France (SP 78), vol. 56, f. 423-428 (cf. aussi Italian States SP 85, vol. 3, f. $154 \mathrm{ss})$.

Cardoini 1621: Andrea Cardoini, «Relatione di Genevra nella quale compendiosamente si ragiona dello stato di quella città, particolarmente dall' anno 1535 che vi fu introdotto il calvinismo et mutato il governo fino al giorno presente» (1621), Genève, Bibliothèque de Genève, ms suppl 28 (cf. aussi ms 1.e.19; ms suppl. 942).

\section{IMPRIMÉS}

Cayet 1599: [Pierre-Victor Palma Cayet], La suite et conclusion de la conférence ténue à Thonon, entre les reverens peres capucins et les ministres de Genève. Où un chacun pourra cognoistre en quelle devotion les desvoyez demeurans six lieus à la ronde de Genève qui estoyent en nombre de onze à douze cents sont venus en procession à Annemace où ils ont faict protestation de la foy catholique apostolique et romaine: où là estoyent pour lors les prieres ordonnées par les catholiques pour la conversion des infidèles, Paris, Denis Binet, 1599. 
Du Ranc 1609: [Jean du Ranc], L'antichrist de Genève où il est clairement monstré que les ministres predicans sont les vrays precurseurs de la beste predite par Daniel, Tournon, Nicolas Colle, 1609.

Raemond 1610: Florimond de Raemond, L'histoire de la naissance, progrez et decadence de l'heresie de ce siècle, Paris, Veuve Guillaume de la Noue, 1610.

De Sales 1634: Charles-Auguste de Sales, Histoire du bien-heureux François de Sales, evesque et prince de Genève [...] par son neveu (Lyon 1634), 2 to., Paris, Louis Vivès, 1885.

Charles de Genève 1653: Charles de Genève, Les Trophées sacrés (ms 1653), éd. mod. par F. Tisserand, 3 vol., Lausanne, Société d'histoire de la Suisse romande, 1976.

Charles de Genève 1657/80: Charles de Genève, Fidèle de Talissieu, Histoire abrégée des missions des pères capucins de Savoye (1657) traduit en français par Fidèle de Talissieu OFM Cap. (1680), éd. moderne, Chambéry, Albert Bottero, 1867.

Locatelli 1691/93: Sebastiano Locatelli, Viaggio di Francia, costumi e qualità di quei paesi (1664-1665), éd. Luigi Monga, Moncalieri, CIRVI, 1990.

Leti 1681: Gregorio Leti, The Present State of Geneva. With a brief description of the City and several changes and alterations it hath been subject to from the first foundation thereof untill this present year 1681, London, William Cademan, 1681.

Leti 1686: Gregorio Leti, Historia genevrina o sia historia della città, e repubblica di Geneva. Cominciando dalla sua prima fondatione fino al presente. Con tutti i Successi, guerre, mutationi di Governi, e di Signorie, et interessi tanto esterni, che interni, con tutti gli avenimenti più riguardevoli, e curiosi. Con un'esatta relatione del suo Stato tanto antico, che moderno, e così spirituale che temporale, e con tutte le Massime più recondite, 5 vol., Amsterdam, Pietro e Abramo van Someren, 1686. 
ANNEXE II

FIGURES 1-4

\section{EMBLEM A IX.}

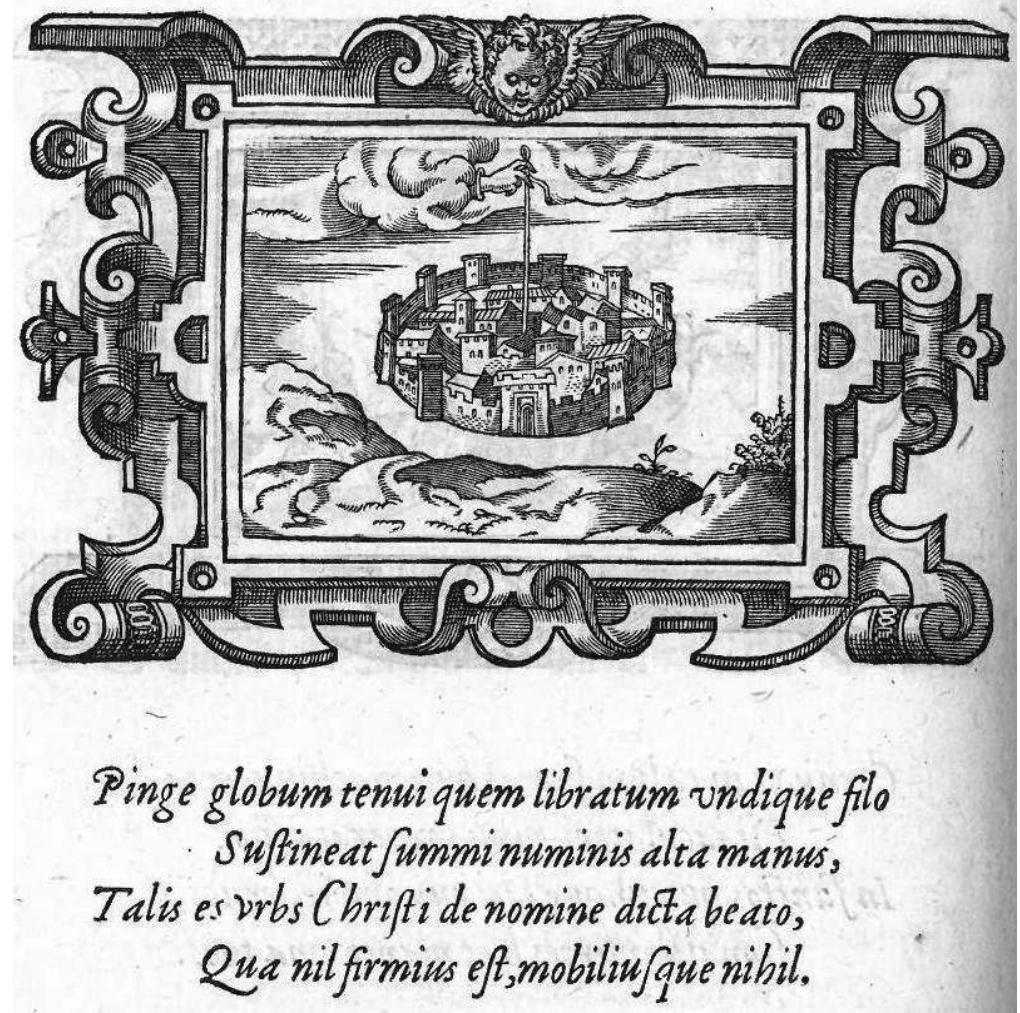

Fig. 1: Théodore de Bèze, «Emblema IX» in: Idem, Icones (Genève 1580) 


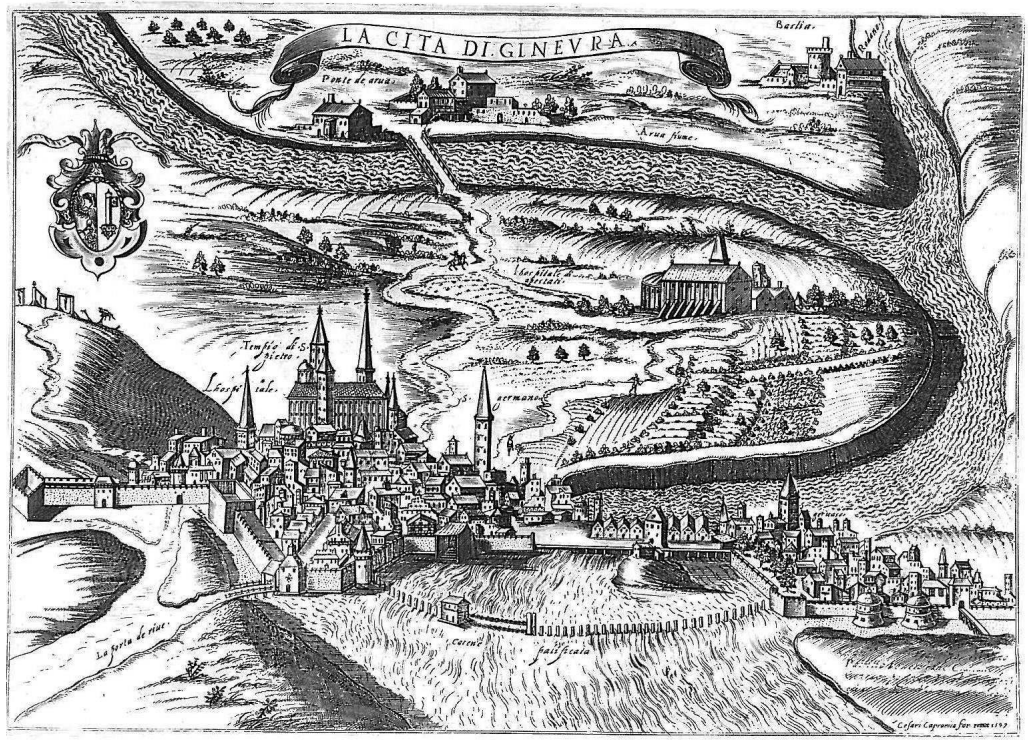

Fig. 2: «La cità di Ginevra» (Cesare Capranica impr., Rome 1597) Bibliothèque de Genève, Centre d'iconographie genevoise (16 M 2)

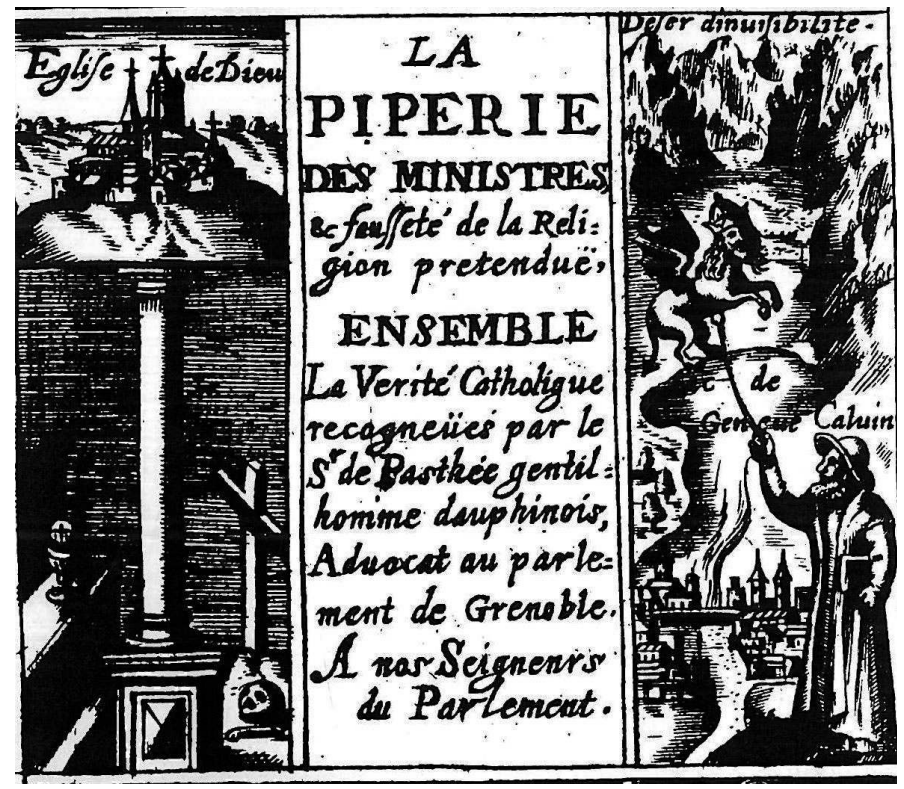

Fig. 3 : Marcelin de Pont-de-Beauvoisin OFMCap., La piperie des ministres et fausseté de la religion prétendue (Lyon 1618, détail du frontispice) 


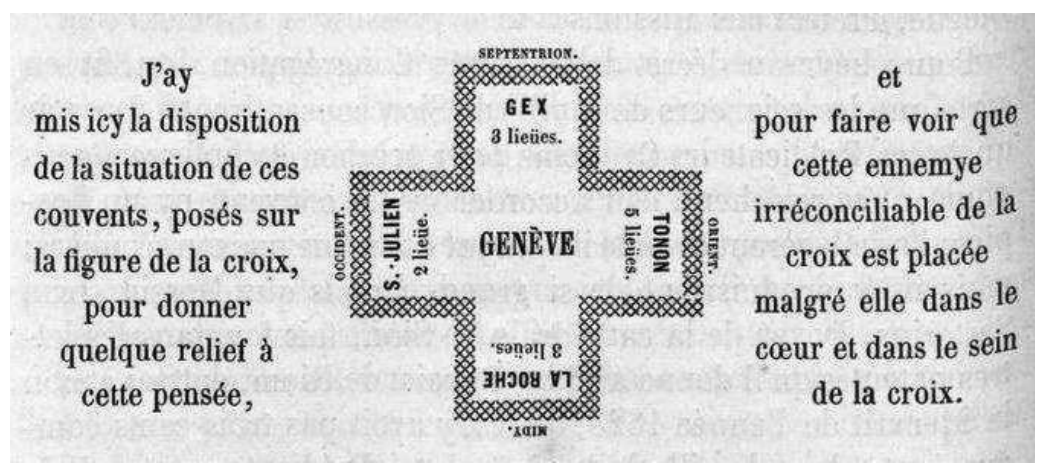

Fig. 4: Charles de Genève, Histoire abrégée des missions des Pères capucins de Savoye (1657/80, éd. Chambéry 1867, détail p. 186) 